Chapter 4 for the Routledge Handbook of Philosophy of Engineering. Diane Michelfelder and Neelke Doorn (eds.). Publication scheduled for 2019. Please do not distribute without permission by the author. Version May 142018.

\title{
Scientific methodology in the engineering sciences
}

MIEKE BOON

Department of Philosophy, University of Twente, PO Box 217, 7500 AE Enschede, The Netherlands. m.boon@utwente.nl

Keywords: Physical phenomena, physical-technological phenomena, scientific concepts, design concepts, scientific models, mathematical modeling, methodology, epistemic aims, epistemic tools, technological artifacts, hypothetical deductive method, B\&K method.

\section{Introduction}

Scientific research in engineering sciences is ubiquitous and nowadays accounts for more research effort than the so-called fundamental or basic natural sciences. Nevertheless, within mainstream philosophy of science relatively little attention has been paid to this type of application oriented scientific research.

The term engineering sciences, however, can be ambiguous and can elicit different understandings. Commonly, the emphasis is on the engineering part of the term, although in this paper the focus will be on the science part - that is, on scientific research in the context of technological applications. This chapter aims to address some salient aspects of the engineering sciences and the methodology, cumulating in a methodology of scientific modeling in the engineering sciences. Section Two aims to characterize the engineering sciences by comparison with scientific research in the basic sciences. A noticeable difference is the role and character of phenomena (see Textbox 1 ), which in the basic sciences serve as aids in discovering and testing theories, while the engineering sciences analyze (physical-technological) phenomena in view of technological functioning or malfunctioning. Scientific research on technological problem-solving and innovation, therefore, is better cast in terms of design-concepts that are based on functional interpretations of phenomena, which is the topic of section Three. Section Four elaborates on the ways in which (physical-technological) phenomena are 
investigated and on the specific character of scientific knowledge for creating or controlling them by means of physical-technological circumstances (see Textbox 1). It will appear that scientific modeling of technological systems is central to the engineering sciences, encompassing both the modeling of physical-technological phenomena in specific physical-technological contexts as well as the modeling of technological artifacts producing specific phenomena. Section Five explains how scientific models of (physical or physical-technological) phenomena are constructed, and proposes a methodology for their scientific modeling (the B\&K method, Figure 1 right-hand side), which is on par with the wellknown hypothetical-deductive methodology (the HD method, also called the empirical cycle, Figure 1). Each section concludes with some remarks about methodology (Textboxes 3, 4, 5, 6) that prepare for the B\&K method of constructing scientific models.

Textbox 1: Clarification of concepts: phenomena and target systems

The term (physical) phenomena refers to existing or non-existent, observable or non-observable, often functionally interpreted physical objects, properties and processes (including regularities and mechanisms). The adjective 'physical' aims to indicate that phenomena exist or potentially exist in the real-world (both nature and technology). The term phenomenon is often used in research-contexts to indicate the 'object of study.' Additionally, the term is used to indicate objects, properties and processes that (supposedly) cause or affect the investigated phenomenon or target-system (see question Q2 in the caption of Figure 1).

The engineering sciences investigate physical-technological phenomena, which can be technological objects, properties and processes, but also technologically produced physical phenomena. For example, a membrane, an electro-magnetic coil and a prosthesis are technological objects, which have specific properties, or which function by means of specific processes. Examples of technologically produced physical phenomena are light, sound, electricity, chemical compounds and all kinds of material properties. Additionally, the term target-system will be used, which is better suited when studying a technological artifact (see Fig 1, Q2).

\section{What is Engineering Science?}

When it comes to the term engineering science philosophers, as well as lay-people, often focus on the engineering part of the term, according to which it is synonymous with engineering and technology (e.g., Vincenti 1990, Meijers ed. 2009). With this interpretation, engineering science becomes synonymous with technological design (with bridges, cars and tools as paradigm examples), 
the development of technological artifacts (such as materials, instruments, devices, industrial processes and other systems), and research towards rational methods of engineering design and development. In this chapter, the emphasis is on the science part, taking the engineering sciences as scientific fields that in many respects resemble basic natural sciences, but with a number of salient differences. ${ }^{1}$

An example of an engineering science is scientific research in the context of chemical engineering. Chemical engineering as an engineering discipline aims at designing and building industrial processes for converting chemicals or raw materials into specific chemical compounds or materials that meet specific functions. Scientific research in the field of chemical engineering typically concerns how to produce a specific chemical compound or material that meets the functional and quality requirements in an effective, reliable, safe and economically feasible manner.

That is why the engineering sciences qua science deal both with scientific knowledge concerning questions such as how a product or technological device can be made that meets specific functional and quality requirements, and how industrial processes for the production of a product or device must be designed and built. These how to questions are usually analyzed in terms of a whole series of questions on mutually coordinated aspects, for example, parallel and serial sub-processes that together form the intended technological configuration or system. Examples of how to questions in chemical engineering concern sub-processes such as: how to produce a specific chemical compound (e.g., a medicine or agricultural chemical); how to achieve the desired chemical conversion or material property and to prevent the undesirable ones (e.g., by using a catalyst or by control of physical-chemical circumstances); how to separate the desired from the undesired compounds (e.g., by distillation or membrane filtration processes); and, how to optimize these sub-processes to reduce costs related to chemicals, energy and waste. Similar types of questions are asked in the context of developing technological devices in material sciences, electrical engineering, mechanical engineering, biomedical engineering and so forth.

In engineering scientific research these questions are phrased as being about phenomena (Textbox 1), which are physical and/or technological objects (e.g., a protein, a membrane, an electrical circuit, a prosthesis), properties (e.g., hydrophobic, magnetic, electrical resistant, elastic) and processes (e.g., chemical production processes, conduction of heat, electro-magnetic processes, transfer of momentum, conversion of energy). Furthermore, a phenomenon often consists of sub-phenomena, which will be explained in more depth in Section Four.

\footnotetext{
${ }^{1}$ See Boon 2011a. In a recent article, I argue that a so-called physics paradigm of science prevents us from recognizing engineering science as a scientific practice. As an alternative, I propose an engineering paradigm of science (Boon 2017b).
} 
Scientific research is crucial to design and development in the sense that scientific research aims at knowledge for the design and development of technology. Here it is important to keep in mind that scientific research aimed at knowledge for is basically motivated by how to questions in technological contexts (see above and Textbox 2).

\section{Textbox 2: Clarification of concepts: Knowledge of phenomena}

Similar to the distinction between real-world and knowledge of that world stressed by Giere et al. (2006), a conceptual distinction is needed between phenomena and knowledge of phenomena. The term knowledge of phenomena will cover different types of epistemic artifacts, such as: descriptions of phenomena (Fig. 1, Q2 and Q5); concepts of phenomena (e.g., 'elasticity', 'conductivity,' 'sound-waves,' 'prosthesis,' 'energy,' 'motor,' 'amplifier'; Fig. 1, Q2); explanations of phenomena (e.g., Fig. 1, Q8 and Q9); measurements of (aspects of) phenomena (Fig. 1, Q6); and, scientific models of phenomena (center Fig. 1).

Additionally, phenomenological laws are the verbal or mathematical representations of reproducible phenomena (Boon 2011b, 2017a). For example, technological devices in an experimental set-up (e.g., Boyle's, Hooke's and Ohm's experiments), produce reproducible physical-technological phenomena that are represented by sentences (e.g., 'there exists an inversely proportional relationship between the pressure and the volume of a gas,' or 'increasing the pressure causes a decrease of the volume') and/or by mathematical equations that relate measured quantities (e.g., P.V=c, F=k.x, and V=I.R).

Engineering sciences aim at knowledge of phenomena that can be used to answer 'how to' questions. As this knowledge is meant to be used in epistemic activities by humans or computers (e.g., regarding questions 'how to' create, design, optimize, etc.) the types of knowledge just mentioned are also called epistemic artifacts. Hence, research aims at knowledge for specific epistemic purposes (Fig. 1, Q3) - for example, a mathematical model of the phenomenon (Fig. 1, center) can be used to make predictive calculations.

Physical-technological phenomena can be created, controlled, optimized etc. by means of physical-technological circumstances, (e.g., temperature, pressure, light, field-strength, chemical composition, material properties, and features of the technological device). Therefore, qualitative or quantitative knowledge of a physical-technological phenomenon (e.g., the model in Fig. 1) must include the role of relevant physical-technological circumstances (see questions Q5 and Q6 in the caption of Figure 1). 
An example is scientific research into membranes. This concerns, for example, the question of how to make a material with which dissolved molecules such as sea salt or proteins can be separated from a fluid, for technological applications such as the production of drinking water from seawater, and medical applications such as kidney dialysis. Another example is research into technological artifacts such as prostheses.

Research topics in the engineering sciences can be motivated in various ways, ranging from pragmatic problem-solving to more fundamental innovative approaches, which are the subject of section Three. In a pragmatic approach, research is usually motivated by specific problems of a technology, and often starts off with a systematic 'trial-and-error' approach in an experimental setup to investigate which physical-technological factors have an effect (see Textbox 2). This pragmatic approach could be characterized as the transition between engineering and science. While a researcher in an engineering context who follows this approach is satisfied with finding a workable solution, scientific research in the engineering sciences usually aims at a more fundamental understanding of the phenomena involved. For example, if the technological problem is to improve the selectivity of a specific membrane, experiments will be set up to examine relevant interrelated physical-technological factors (e.g., the chemical structure of the membrane, selectivity, efficiency, permeability, fouling, strength, membrane thickness, pore size and size distribution, osmotic pressure and shear stress), and investigate how changes of the material properties affect these factors, vice versa. Also, existing scientific understanding of these factors will be used to design the experimental set-up and to interpret the measurements, which results in increasing scientific understanding of the specific physical-technological phenomena (i.e., materials, properties and processes) involved.

Summing up, similarity between the engineering sciences and the basic natural sciences consists in aiming to understand phenomena scientifically. In both cases, this can be done in a systematic 'trial and error' manner and in a more fundamental way, which often requires more innovative scientific approaches (section Three). Both develop and use technological instruments for experimentally generating and investigating the phenomena. Also, both adopt systematic approaches and scientific methodology to check the results. Additionally, both aim at scientific modeling, the results of which are published in scientific articles. A salient difference, however, is due to the application-context of designing and developing technological artifacts. Therefore, in the engineering sciences, the epistemic aim of investigating phenomena and of developing technological instruments is not firstly to discover or test scientific theories, but rather, knowledge for how a functionally relevant phenomenon is created, produced, improved, controlled, manipulated, prevented or optimized through physical-technological circumstances (Textbox 2). 
This application context of aiming at knowledge for reasoning about phenomena in specific physicaltechnological circumstances, i.e., knowledge that enables and guides epistemic agents' reasoning about a phenomenon in physical-technological circumstances actually implies that the term 'phenomenon' has a slightly different meaning in the engineering sciences (Textbox 1 ) - and thus also differs from how the philosophy of science usually thinks about phenomena (e.g. Hacking 1983, Bogen\&Woodward 1999, Bailer-Jones 2009). As already said, in basic natural sciences, phenomena are aids to research. Conversely, in engineering sciences phenomena are part of the goals of research. Furthermore, phenomena are interpreted in terms of their physical features and in terms of their technologically relevant (dys)function. They have a dual nature so to speak (Kroes 1998, 2010; Weber et al. 2013). It is also important to recognize that in the basic natural sciences, scientific understanding of phenomena is usually detached from technology - phenomena are often presented as somehow 'free-floating' in nowhere. Contrariwise, in the engineering sciences, phenomena are firstly understood as 'embedded' in physical and physical-technological circumstances.

Textbox 3: Methodology: Towards the B\&K method of (re-)constructing scientific models - from technological function to phenomena.

How do the indicated similarities and differences between the engineering sciences and the basic natural sciences affect methodology? A few aspects can already be pointed out. To begin with, scientific research in the engineering sciences is motivated by a technological application context, which requires a specific methodological approach when specifying the research question (Fig. 1, Q1). The research question relates to, say, the proper (or improper) functioning of technological artifacts, which is understood in terms of physical-technological phenomena (Fig. 1, Q2). Accordingly, scientific research aims at practical and scientific understanding of how these phenomena are technologically produced (or created, prevented, controlled, improved, manipulated, optimized, affected, etc.), that is, at the construction of the scientific model of the phenomenon (center of Fig. 1, right-hand side), which usually goes hand-in-hand with the development of technological devices and procedures (for their actual production, or their creation, prevention, control, improvement, etc.), and often also with the development of computer programs for simulating them in the development and design phase.

\section{Design-concepts in the engineering sciences}


Innovative approaches often start with a new design-concept for a specific technological function. Whereas a more pragmatic approach aims to improve the performance of an already existing technology, not fundamentally changing the physical-technological way in which the specific technological function is achieved, a more innovative approach starts from asking whether the same technological function can perhaps be achieved in a different way. A well-known strategy is biomimicry or bioinspired approaches, where scientists learn from nature by looking how specific functions are generated in biological systems (e.g., how natural membranes work, how photosynthesis produces energy, or how musculoskeletal systems work). An innovative strategy can aim at solving existing problems, but also at new innovative opportunities that no one has ever thought of. Creative minds look at salient phenomena from a functional perspective, thereby inventing new design-concepts for new ways of achieving technological functions. Let us look at a few examples that elaborate on technologies for the production of sugar.

When one learns chemistry in high-school and sees a stoichiometric equation of how glucose (sugar) is produced from carbon dioxide and water, vice versa: $6 \mathrm{CO}_{2}(\mathrm{~g})+6 \mathrm{H}_{2} \mathrm{O}(\mathrm{I})=\mathrm{C}_{6} \mathrm{H}_{12} \mathrm{O}_{6}(\mathrm{aq})+6 \mathrm{O}_{2}(\mathrm{~g})$, one may tend to believe that this is how glucose can be produced industrially. This thought is a rudimentary design-concept. However, this chemical conversion is not effective, nor economically feasible, and in any case impossible to do in a simple way.

What seems feasible, is another design-concept in which carbon dioxide is converted by means of sunlight into glucose or other 'energy-rich' compounds such as hydrogen gas or ethanol, by using natural catalysts. This design-concept is attractive because it can help to solve the problem of storing solar energy (e.g., as produced by solar panels on roofs). 'Clean' energy-rich compounds thus produced can then be supplied to already existing technological systems that produce electrical energy or heat by converting these compounds back to $\mathrm{CO}_{2}$ (carbon dioxide) and $\mathrm{H}_{2} \mathrm{O}$ (water). Hence, the general, 'to be realized' design-concept is artificial photo-synthesis to harness solar energy (e.g., Pandit et al. 2006, Ong et al. 2018). Yet, this general design-concept needs to be made more concrete - it needs to be translated into design-concepts of realizable technological systems for fulfilling the technological function of artificial photo-synthesis.

The development of an artificial photosynthesis technology faces major challenges. For instance, how to create an effective, robust, etc. (bio-inspired) catalyst for $\mathrm{CO}_{2}$-reduction by sunlight; how to create a carrier for the catalyst; and, how to create a large surface-area at which the sun shines and artificial photosynthesis takes place. One design-concept to meet these challenges is to create a high surfacearea on which sunlight can shine and artificial photosynthesis can take place, by means of a reactor consisting of shallow micro-channels. In this so-called opto-fluidic micro-reactor a membrane separates the $\mathrm{CO}_{2}$-containing gas-phase from the water-phase flowing through the micro-channels. 
The membrane is also the carrier for the catalyst that promotes photosynthesis. Hence, the membrane has three functions: it is the surface at which photosynthesis takes place; it selectively allows transport of specific molecules across the membrane; and it separates the water-flow that transports the energy-rich products from the gas-flow that transports $\mathrm{CO}_{2}$ (Huang et.al. 2018).

Design-concepts also concern the bio-inspired catalyst, which are inorganic molecules called artificial light-harvesting antennas. Scientific research firstly aims at finding molecules that transfer charge (electrons), and next, to understand the properties of these light-harvesting antennas - for instance, in order to design molecular structures that have the highest possible electron-transfer efficiency (e.g. Uosaki et al. 1997; Gust et al 2001; Dubey et al. 2016).

Summing up, the engineering sciences typically start from problems regarding specific functions or dysfunctions. Crucially, scientific researchers aim to understand these problems in terms of physicaltechnological phenomena (i.e., objects, properties and processes). From an engineering science perspective, physical phenomena can be naturally or technologically generated - in this latter case, we call them physical-technological phenomena. Also, phenomena can cause the functioning but also the malfunctioning of a technology. The engineering sciences, therefore, can be understood as aimed at the creation and control of physical-technological phenomena through technological means. The epistemic task is scientific knowledge for how to do this. This knowledge concerns the physicaltechnological phenomena, including the technological devices that can be understood in terms of desired functional and undesired dysfunctional phenomena. 
Textbox 4: Methodology: Toward the B\&K method of (re-)constructing scientific models - from phenomena to design-concepts.

The examples in this section show how scientific researchers in the engineering sciences are innovative by inventing new design-concepts for specific functions, which often start at a very general level. The challenge is to translate these into more concrete design-concepts, for instance, a concept of a technological system that actually does the job. This is where scientific research starts, and also, where many brilliant ideas ultimately fail (Fig. 1, Q1 and Q2 cover this dynamics). The presented examples also show that although a general design-concept is often inspired by functional aspects elsewhere (e.g., bio-mimicking or bio-inspired technology), the functional parts (e.g., natural membranes, micro-channels and catalysts in natural photosynthesis and musculoskeletal systems) usually are not literally reproduced in the technology. Instead, the task of the engineering sciences is to find out how functional objects, properties and processes that are not yet existent, but that are presented as design-concepts, can be created by 'artificial' technological means. Importantly, this also implies that the engineering sciences not only aim at utilizing existing phenomena, but also at creating physical-technological phenomena that do not exist as yet (Boon 2012, 2017a).

\section{Scientific knowledge for creating or controlling physical-technological phenomena}

In scientific research that aims at (technologically) creating or developing the function indicated by the design-concept, knowledge of physical-technological phenomena can play several roles.

Researchers may aim to technologically mimic the physical phenomena, which requires scientific research that gets them from a mere description of the phenomenon to a scientific model of it that allows for its technological reproduction in a more or a less literal fashion (Fig. 1, right-hand side). For instance, researchers may try to technologically create chlorophyll molecules in order to develop artificial photosynthesis technology, but often these kinds of approaches are not feasible.

Nevertheless, in-depth scientific understanding of the phenomenon (e.g., knowledge of the chemical structure of the chlorophyll molecule, its bio-chemical mechanism and its energetic efficiency) is of crucial importance (Pandit et al. 2006, Dau et al. 2017). As was illustrated above, rather than literally mimicking, researchers often only aim at utilizing the underlying 'physical principles', which involves the interpretation of the functioning of the phenomenon at a more abstract conceptual level. For example, scientific research that aims at developing 'artificial photosynthesis' builds on knowledge of 
the phenomenon 'electron-transfer by chlorophyll molecules' as a principle by which solar energy is harnessed. This knowledge is used to develop artificial inorganic molecules that can do the electrontransfer in a 'similar' fashion (e.g., Uosaki et al. 1997; Dubey et al. 2016).

Another role played by knowledge of physical phenomena in the (technological) creation, control or development of the function indicated by the design-concept is based on a modular approach that is made possible by thinking in terms of phenomena. In the engineering sciences - technologically produced-physical-technological phenomena are conceived as physical building-blocks for physically creating, managing and developing technological functions. Scientific knowledge of physical-technological phenomena, therefore, consists of epistemic building-blocks for the design and development of a specific technological function. Hence, modular knowledge of phenomena enables and guides the understanding of a design-concept in terms of mutually interacting phenomena - also indicated by higher-level/lower-level, horizontal/vertical and parallel/sequential phenomena as well as networks thereof. Knowledge of phenomena is usually represented through scientific models (center of Fig. 1, right-hand side), and the modular approach allows for the construction of so-called multi-scale models. Hence, this scientific approach prompts scientific researchers to analyze a specific functional phenomenon in terms of a combination of interacting phenomena.

In chemical engineering, for example, the full chemical process for, say, producing a chemical compound, is analyzed in terms of more basic phenomena such as: desired and undesirable chemical reactions in producing the intended chemical compound; the transport of liquids, gases and solids within the device; the transport of chemical compounds by means of fluid flow and diffusion processes within the fluid; the transport of heat by convection and conduction, and other physical processes such as absorption, dissolution, ionization, precipitation, vaporization and crystallization. ${ }^{2}$ A crucial strategy in the engineering sciences is to analyze physical phenomena in nature and as produced by technological devices in terms of more basic physical phenomena. Importantly, although physical phenomena are referred to as individual physical entities, they should not be understood as entities that exist independently. Many physical phenomena are technologically generated and are in that sense dependent on physical and technological circumstances. Examples of technologically produced phenomena -i.e., physical-technological phenomena- are electrical currents, electro-magnetic waves, piezo-electricity, super-conductivity and selective permeability of membranes. Other 'naturally occurring' phenomena only manifest at specific, controlled physicaltechnological conditions (e.g., catalyzed chemical reactions, boiling-point of a substance, standing

\footnotetext{
${ }^{2}$ e.g. Westerterp et al. (1984). See Schneider et al. 2003 for an accessible example on modeling interacting phenomena, and see Boon 2008 for an explanation of these types of diagrammatic models.
} 
sound-waves, electron beams and Röntgen rays). Nevertheless, in order to be recognized as a phenomenon at all, it must manifest itself in a reproducible manner (Bogen and Woodward 1988). From a technological point of view, it is precisely these characteristics of phenomena that make them technologically interesting and, moreover, manageable for scientific research.

Therefore, different from the more naive view of independently existing phenomena as we often see in textbooks, it is stressed here that technologically relevant phenomena need to be understood as in a relationship with relevant physical and technological circumstances. The task of scientific research in the engineering sciences is then to generate scientific knowledge of physical or physicaltechnological phenomena significant to specific technological problems or functions that take into account the role of relevant physical-technological circumstances (see Textbox 2).

Furthermore, in the engineering sciences, descriptions, conceptions, explanations, measurements and scientific models of physical or physical-technological phenomena are closely related to designconcepts, but this relationship is multi-layered. As has been illustrated above, knowledge of salient physical or physical-technological phenomena often inspires a design-concept when the observed or purported phenomenon is interpreted in terms of a specific function. Photosynthesis (i.e., a purported, non-observable phenomenon that causes observed physical or physical-technological phenomena) is interpreted in terms of the function 'energy-production'; while the human musculoskeletal system can be interpreted in terms of 'mechanical movement and support of the body,' and next, as a design-concept for the exoskeleton. Making these creative leaps between 'knowledge of a phenomenon' to 'function of the phenomenon' involves an act of 'seeing as' by an engineering mind that asks how the phenomenon can be practically and technologically utilized. 
Textbox 5: Methodology: Toward the B\&K method of (re-)constructing scientific models - The production of scientific knowledge

Scientific research can produce different types of scientific knowledge about a phenomenon under study. An elementary form of scientific knowledge of a physical phenomenon is observed regularities in measurements, which are represented by means of phenomenological laws or mathematical equations that describe causal relations, correlations or statistical relationships between observed and measured variables (also see Textbox 2). Scientific models of the phenomenon are a more sophisticated form of scientific knowledge. These models can be mathematical, causal-mechanistic or diagrammatic models, or a combination of these, which includes multi-scale and network models (Fig. 1, Q4). In the engineering sciences, scientific knowledge production is also guided by epistemic purposes in specific technological application contexts (Fig. 1, Q1), while it is confined by available knowledge (Fig. 1, Q8) and (experimentally or computationally generated) data about the phenomenon (Fig. 1, Q5).

\section{Constructing and reconstructing scientific models: the B\&K method next to the HD method}

In the engineering sciences, education in mathematical modeling of phenomena and technological systems is strongly developed. Several mathematical approaches can be distinguished (e.g., Dym 1980/2004). The most rudimentary approach starts from reproducibly measured, quantitative datasets and aims to find mathematical patterns or structures in them, represented by an algorithm, such as linear or exponential equations (considered phenomenological laws such as Boyle's law or Hooke's law) or a set of equations that forms a mathematical model for a specific phenomenon or system. Another approach is to translate a conceived mechanism into a mathematical model. Examples are the Lotka-Volterra model of predator-prey dynamics and the Maxwell-Boltzmann model for describing the mean distribution of non-interacting particles at random velocity in statistical mechanics. Yet another well-known approach is to interpret a system or phenomenon as being a specific type (e.g., a harmonic oscillator as being a Newtonian system), and then construct a mathematical model by derivation from the fundamental (axiomatic) theory (i.e., fundamental theories such as Newtonian mechanics, quantum mechanics, fluid mechanics, thermodynamics and electro-magnetism) for this (idealized) type of system. In scientific practices, mathematical models 
are often constructed through a combination of these approaches, including the construction of diagrammatic models (Boon 2008).

Surprisingly, however, engineering science education often pays little attention to non-mathematical ways of scientific modeling. Rather, the hypothetical-deductive method (e.g., as in Hempel 1966) is usually put forward as a methodology to test explanations of observed phenomena (i.e., the hypothesis in the HD method, Fig. 1, left-hand side), while assuming that the way in which hypotheses come about is either a highly creative process, or rather superficially by inductive inference, as in 'all swans are white.' Hence, although HD reasoning definitely plays a role in testing hypotheses, it hardly provides guidance to how scientific models that explain physical-technological phenomena (e.g., in terms of non-observable phenomena and physical-technological circumstances) are put together.

This defective representation of the construction of scientific models is a serious shortcoming of textbooks in the engineering sciences. This shortage is also present in the Philosophy of Science and in the Philosophy of Engineering. ${ }^{3}$ Another deficiency due to not teaching students how scientific models for phenomena are constructed is the way in which explanatory or causal-mechanistic models (e.g., the model of DNA) are usually presented in textbooks. These models suggest first that the phenomenon has been observed in science; second, that the phenomenon exists independent of physical or physical-technological conditions; and, thirdly, related to the above, it is usually not reported how the phenomenon is actually detected, nor, how it is produced or manipulated by means of technological devices - that is, it is often not made clear how the purported (nonobservable) phenomenon is connected with the tangible world. In short, explaining scientific research in terms of the HD-method only, does not serve to understand how scientific knowledge for specific epistemic task (such as those described in this chapter) is produced and utilized.

Since Morgan \& Morrison's (1999) collection Models as Mediators it is generally accepted that scientific models often are autonomous epistemic artifacts, rather than merely derived from fundamental theories, as was assumed in the semantic view of theories. ${ }^{4}$ Accordingly, Boumans (1999) argues that scientific models consist of several 'ingredients,' and that the way in which these ingredients are put together already provides quite a bit of the justification of the model. Knuuttila and I have elaborated on this idea, resulting in a more specific list of elements that usually play a role in building scientific models. Moreover, we argue that researchers can actually use scientific models

\footnotetext{
${ }^{3}$ Some notable exceptions are: Magnani \& Bertolotti (eds. 2017), Nersessian (2009 a, b, c, 2012), Sterrett (2002, 2006, 2009, 2014, 2017).

${ }^{4}$ Clear accounts of the semantic view can be found in Suppe (1989) and Giere (2010). Cartwright $(1983,1989$, 1991) and Morgan\&Morrison (1999) have convincingly argued that this view is very restricted on the role of models in science.
} 
for epistemic tasks because of how models are built, rather than models being first and foremost objective, human-independent representations of their real-world target (Boon\&Knuuttila 2009; Knuuttila\&Boon 2011). ${ }^{5}$

Textbox 6: Methodology: Toward the B\&K method of (re-)constructing scientific models

The B\&K method for (re-)constructing scientific models considers scientific models for physical or physical-technological phenomena. It suggests that a model can be analyzed in terms of several elements. The B\&K method consists of ten questions to systematically analyze these elements (see caption Fig 1, questions Q1-10). Thereby, it allows the analysis of the elements that play a role in constructing a scientific model or in reconstructing how a model is put together. Importantly, this list is not intended to present an algorithm by means of which the scientific model can be derived - as seems to be the case in mathematical modeling based on fundamental theories. Instead, the B\&K method guides the process of gathering relevant information including different types of scientific knowledge- about the phenomenon, which researchers need to fit together into a scientific model that suits their epistemic purpose(s). Vice versa, the $B \& K$ method guides the reconstruction of scientific models (e.g., models presented in scientific articles or research projects) by systematically retrieving the elements that went into them. Knuuttila\&Boon $(2011)^{5}$ present an elaborate example in which we reconstruct Sadi Carnot's model of the ideal heat-engine through this method. Here, I will only present an outline of the B\&K method.

\footnotetext{
${ }^{5}$ Based on teaching the $B \& K$ method in analyzing scientific articles and research projects in engineering science education, the original list of eight elements in Knuuttila\&Boon (2011) has been expanded with 'problemcontext' (Q1) and 'hypotheses' (Q9), resulting in the ten questions listed in the caption of Figure 1. Also, in teaching, I have started to call it the B\&K method for (re-)constructing scientific models (Fig 1, right-hand side and caption), as an alternative to the traditional HD-method (Fig 1, left-hand side).
} 
HD method in natural sciences

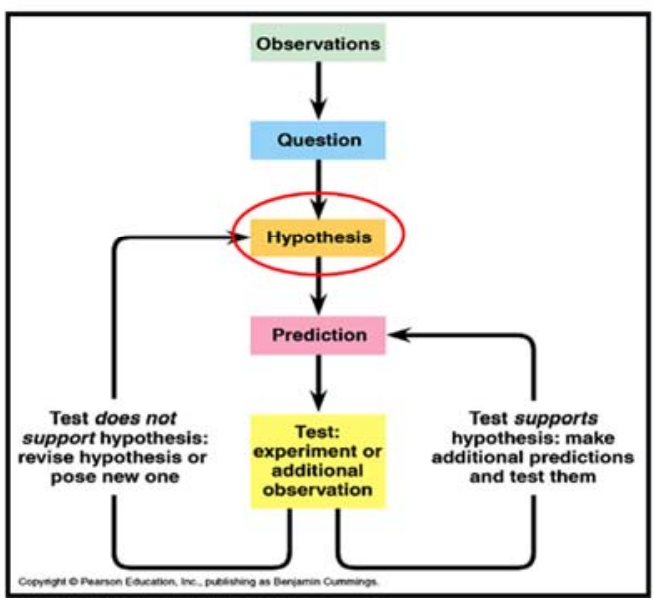

$B \& K$ method for (re)constructing scientific models of phenomena

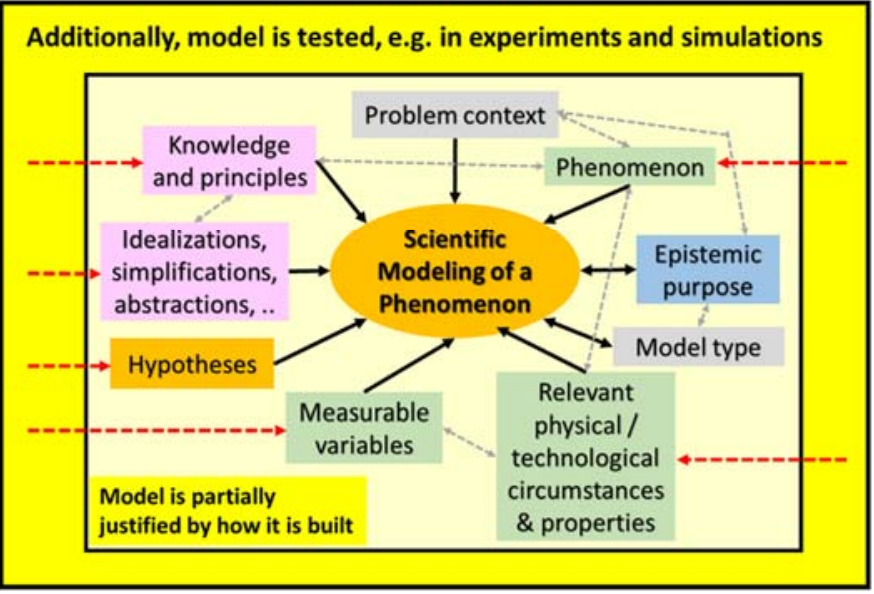

Figure 1 (right-hand side): The B\&K method consists of ten 'what is/are?' questions (Q1-10) to systematically determine the concrete elements that are 'built-in' the scientific model: (Q1) Problem context? (Q2) Targetsystem or physical-technological phenomenon (P) for which the model is constructed? (Q3) Intended epistemic function(s) of the model? (Q4) Model type? (Q5) Relevant (physical and/or technical) circumstances and properties (i.e., those that affect the phenomenon)? (Q6) Measurable (physical-technological) variables (i.e., by which variables is a non-observable phenomenon connected to the tangible world, or, by which variables is the phenomenon or target-system affected)? (Q7) Idealizations, simplifications and abstractions? (Q8) Knowledge (theoretical knowledge, knowledge of sub-phenomena, phenomenological laws, empirical knowledge) and theoretical principles used in the construction of the model? (Q9) Hypotheses (e.g., new concepts and explanations) 'built-in' the model? (Q10) Justification and testing of the model? The red arrows indicate elements that can be modified when testing and improving the model. The yellow square surrounding the modeling process indicates the testing phase in Q10.

Basically, the collection of questions (Q1-10) that form the heart of the B\&K method, account for several important characteristics of scientific modeling in the engineering sciences. The first characteristic is that the target-system or physical-technological phenomenon of interest (Q2) is often investigated in a broader, more complex problem-context (Q1), and that the scientific model of the phenomenon must be such that it allows for epistemic uses in that problem-context (Q3). Thus, the intended epistemic uses may call for a specific type of model (Q4). For instance, if researchers aim at investigating the dynamic behavior of the system in computer simulations, one needs a mathematical model, but if researchers aim at knowledge of how to create a specific function, a (causal-)mechanistic or network model is needed. Hence, what the scientific model of the phenomenon 'looks like' is partially determined by this context. 
A second characteristic is that researchers usually aim to investigate and to scientifically model a functional or dysfunctional phenomenon --or target-system-- in isolation. Hence, whereas the problem-context (Q1) refers to the function of a technological artifact, the specific research usually focuses on specific (sub-)phenomena or parts of the target-system (Q2), for instance, (subIphenomena or parts that are held causally responsible for the (mal-)functioning of the technological artifact, or on discovered (sub-)phenomena found in measured data (Boon 2012).

A third characteristic is that the construction of scientific models builds on existing knowledge (Q5 and Q8). To begin with, scientific models are therefore considered hubs for bringing together and integrating relevant information and scientific knowledge about the phenomenon. Gathering knowledge about the phenomenon is not only based on textbook knowledge and scientific literature, but is also often produced in an interaction with developing and using experimental (and computer) models (Nersessian\&Patton 2009b,c). The B\&K method distinguishes two types of knowledge, although there may be overlap. Q5 focuses on knowledge about the physical-technological circumstances affecting the phenomenon, while Q8 focuses on all kinds of scientific knowledge about the phenomenon or target-system P - including knowledge of 'lower-level' phenomena that are held causally responsible for (properties and functioning of) the phenomenon or target-system P. Clearly, the construction of the model depends on available knowledge, i.e., the sophistication of a scientific field. Yet, the phenomenon or target-system do not provide information about what should be included in the model. Instead, the expertise of scientific researchers is crucial for deciding which information and scientific knowledge about the phenomenon is relevant to the model (and in view of the intended epistemic aim of the model, Q3).

A fourth characteristic is that the scientific model must account for how (aspects of) the phenomenon or target-system are latched to the tangible world - how are these aspects detected in measurements (Q6) and how is the phenomenon or target-system affected by physical-technological conditions (Q5). It is important to notice that not all relevant conditions (Q5) are actually measurable (Q6). Pointing out what is measurable, indicates the confines within which a scientific model is built. Additionally, the measurable variables and parameters determine the testability of the model (Q10). Aspects in the model that cannot somehow be latched to (imaginable) measurable or observable data do not make sense, which is why in scientific research scientific modeling often goes hand-inhand with the development of experimental and measurement procedures (Boon 2017a), and also, why the construction of scientific models is confined by measurement procedures (even if only conceivable and not yet actual) and available data.

Fifth, the B\&K method points out several elements common to model-building. Usually, modeling involves idealizations, abstractions and simplifications (Q7). Different from the naive idea that 
models are idealization of something else -implying that idealizations etc. are made on the basis of some kind of unpolished picture of the non-observable(!) phenomenon or aspects of the targetsystem-, idealizations, abstractions and simplifications rather concern the heterogeneous bits of information and scientific knowledge that go into the model (Q5 and Q8). Making the decisions on 'what goes in and what not,' involves, for instance, a trade-off between simplicity and exactness, which needs to be assessed in view of the epistemic purpose of the model (Q3). Another important aspect of model-building is that hypotheses of all kinds may be built into the model (Q9). These hypotheses are sometimes 'real discoveries' concerning explanations of the phenomena, but often just 'small insights' concerning assumptions on the relevance of physical and technological circumstances (Q5) or sub-processes (Q8), or the assumptions made in simplifications and idealizations (Q7).

Finally, a crucial characteristic of modeling is that the model needs to be justified and tested (Q10). As said, part of the justification is already done through justified decisions by researchers along the lines of the questions in the B\&K method. Additionally, the model can be tested by comparison between model-predictions and outcomes of experiments or computer-simulations - which is the type of justification basically assumed in the semantic view (e.g., Suppe 1989, Giere 2010). Negative test-results will require reconsidering justification of the elements built into the model.

It is not claimed that every scientific model necessarily entails these ten elements. Rather, the B\&K method aims at providing a schema that assist the (re)construction of scientific models of phenomena, similar to the way in which the schema of the hypothetical-deductive method assists in recognizing elements playing a role in the dynamics of scientific research that aims at theories.

Lastly, the B\&K method provides important insights into the general character of scientific models in the engineering sciences. In the philosophy of science, it is widely held that models represent their target (the physical or physical-technological phenomenon), and debate concerns what constitutes this representational relationship. As said, scientific models presented in the basic sciences seem to suggest that there is a similarity relationship as in a drawing or a photo. Yet, this suggestion is epistemologically unsound. Furthermore, in the philosophy of science it is often assumed that models are mere non-linguistic entities (mathematical structures, pictures, diagrams), whereas the B\&K method emphasizes that scientific models also entail descriptive, numerical and theoretical, empirical and practical 'background' information relevant to the phenomenon or target-system, and also that this content is critical to epistemic uses of scientific models. Constructing and understanding scientific models in the engineering sciences, therefore, crucially involves the elements pointed out in the B\&K method. 


\section{Acknowledgements}

This work is financed by an Aspasia grant (2012-2017) from the Dutch National Science Foundation (NWO) for the project "Philosophy of Science for the Engineering Sciences." A draft of this article was presented at the Models and Simulations conference (MS8) in 2018 at the University of South Carolina, and I wish to thank the organizers and the audience for helpful suggestions. I also wish to thank Henk Procee, Neelke Doorn, Diane Michelfelder and Thendral Govindaraj for their constructive suggestions. Thanks to Claire Neesham for reading the paper as native English speaker.

\section{Further Reading}

- Hans Radder (2003) edited a collection The Philosophy of Scientific Eexperimentation, which presents state of the art philosophical studies of the role of technological instruments in scientific experimentation. In a review article about this collection, I distinguish between three different roles of technological instruments: to measure, to model and to manufacture (Boon 2004).

- Sven Ove Hansson (2015), in a similar fashion, has edited The Role of Technology in Science, in which already more emphasis is put on the development of the technology next to its role in testing scientific theories.

- Wibo Houkes and Pieter Vermaas (2010), in Technical Functions: On the Use and Design of Artifacts have addressed the issue of technological functions from the perspective of the design of technological artifacts, in which - different from the methodology presented in this chapter, the role of the users of artifacts is pivotal. This work is discussed by the authors and reviewers in Weber et al. (2013).

- Anthonie Meijers (2009) has edited a handbook in the philosophy of science: Philosophy of Technology and Engineering Sciences. This handbook touches on many important issues in engineering and engineering sciences. One of the ideas put forward is the notion of technological knowledge as an autonomous kind of knowledge, which is critically discussed in Boon (2011a).

- Mary Morgan and Margaret Morrison (1999) have edited the collection Models as Mediators, which has played a pivotal role in attention to the role of models and modeling in scientific research.

- Daniella Bailer-Jones' (2009) monograph presents a comprehensive account of Scientific Models in Philosophy of Science.

\section{References}


Bailer-Jones, D. M. (2009). Scientific Models in Philosophy of Science. Pittsburgh: Pittsburgh University Press.

Bogen, J. (2011). 'Saving the phenomena' and saving the phenomena. Synthese, 182(1), 7-22. doi:10.1007/s11229-009-9619-4

Bogen, J., \& Woodward, J. (1988). Saving the Phenomena. The Philosophical Review, 97(3), 303-352. doi:10.2307/2185445

Boon, M. (2004). Technological instruments in scientific experimentation. International Studies in the Philosophy of Science, 18(2\&3), 221-230.

Boon, M. (2008). Diagrammatic Models in the Engineering Sciences. Foundations of Science, 13(2), 127-142. doi:10.1007/s10699-008-9122-2.

Boon, M., \& Knuuttila, T. (2009). Models as Epistemic Tools in Engineering Sciences: A Pragmatic Approach. In A. Meijers (Ed.), Philosophy of Technology and Engineering Sciences. Handbook of the Philosophy of Science (Vol. 9, pp. 687-720): Elsevier/North-Holland.

Boon, M. (2011a). In Defense of Engineering Sciences: On the Epistemological Relations Between Science and Technology. Techné: Research in Philosophy and Technology, 15(1), 49-71.

Boon, M. (2011b). Two Styles of Reasoning in Scientific Practices: Experimental and Mathematical Traditions. International Studies in the Philosophy of Science, 25(3), 255-278.

Boon, M. (2012). Scientific Concepts in the Engineering Sciences: Epistemic Tools for Creating and Intervening with Phenomena. In U. Feest \& F. Steinle (Eds.), Scientific Concepts and Investigative Practice (pp. 219 - 243). Berlin: De Gruyter.

Boon, M. (2017a). Measurements in the Engineering Sciences: An Epistemology of Producing Knowledge of Physical Phenomena. In N. Mößner \& A. Nordmann (Eds.), Reasoning in Measurement (pp. 203-219). London and New York: Routledge.

Boon, M. (2017b). An Engineering Paradigm in the Biomedical Sciences: Knowledge as Epistemic Tool. Progress in Biophysics and Molecular Biology, 129, 25-39. doi:https://doi.org/10.1016/j.pbiomolbio.2017.04.001

Boumans, M. (1999). Built-in Justification. In M. S. Morgan \& M. Morrison (Eds.), Models as Mediators Perspectives on Natural and Social Science (pp. 66-96). Cambridge: Cambridge University Press.

Cartwright, N. (1983). How the Laws of Physics Lie. Oxford, Clarendon Press, Oxford University Press.

Cartwright, N. (1989). Nature's Capacities and their Measurement. Oxford: Clarendon Press, Oxford University Press.

Cartwright, N. (1999). The Dappled World. A Study of the Boundaries of Science, Cambridge University Press.

Dau, H., Fujita, E., \& Sun, L. C. (2017). Artificial Photosynthesis: Beyond Mimicking Nature. Chemsuschem, 10(22), 4228-4235. doi:10.1002/cssc.201702106 
Dubey, R. K., Inan, D., Sengupta, S., Sudholter, E. J. R., Grozema, F. C., \& Jager, W. F. (2016). Tunable and Highly Efficient Light-harvesting Antenna Systems based on 1,7-perylene-3,4,9,10-tetracarboxylic acid Derivatives. Chemical Science, 7(6), 3517-3532. doi:10.1039/c6sc00386a

Dym, C.L. (1980, 2nd edition 2004). Principles of Mathematical Modeling. Amsterdam: Elsevier Academic Press.

Giere, R. N. (2010). An Agent-based Conception of Models and Scientific Representation. Synthese, 172(2), 269281. doi:10.1007/s11229-009-9506-z

Giere, R. N., Bickle, J., \& Mauldin, R. (2006/1979). Understanding Scientific Reasoning (5th edition ed.). Belmont, CA Thomson/Wadsworth.

Gust, D., Moore, T. A., \& Moore, A. L. (2001). Mimicking Photosynthetic Solar Energy Transduction. Accounts of Chemical Research, 34(1), 40-48. doi:10.1021/ar9801301

Hansson, S. O. (Ed.) (2015). The Role of Technology in Science: Philosophical Perspectives (18 ed.). Dordrecht/Heidelberg: Springer.

Hempel, C. G. (1966). Philosophy of Natural Science: Englewood Cliffs, N.J. : Prentice-Hall. https://www.nature.com/articles/nmeth.2651\#supplementary-information

Huang, X. W., Wang, J. C., Li, T. H., Wang, J. M., Xu, M., Yu, W. X., .. . Zhang, X. M. (2018). Review on Optofluidic Microreactors for Artificial Photosynthesis. Beilstein Journal of Nanotechnology, 9(1), 30-41. doi:10.3762/bjnano.9.5

Knuuttila, T.T., \& Boon, M. (2011). How do Models give us Knowledge? The case of Carnot's ideal Heat Engine. European Journal for Philosophy of Science, 1(3), 309-334. doi:10.1007/s13194-011-0029-3

Kroes, P. (1998). Technological Explanations: The Relation between Structure and Function of Technological Objects. Techné: Research in Philosophy and Technology, 3(3), 124-134. doi:10.5840/techne19983325

Kroes, P. (2010). Engineering and the Dual Nature of Technical Artifacts. Cambridge Journal of Economics, 34(1), 51-62. doi:10.1093/cje/bep019

Magnani L.; T. Bertolotti (eds. 2017). Springer Handbook of Model-Based-Science. Springer.

Meijers, A. (Ed.) (2009). Philosophy of technology and engineering sciences. Handbook of the Philosophy of Science (Vol. 9): Elsevier/North-Holland.

Morgan, M. S., \& Morrison, M. (Eds.). (1999). Models as Mediators - Perspectives on Natural and Social Science. Cambridge: Cambridge University Press.

Nersessian, N. J. (2009a). Creating Scientific Concepts. Cambridge, MA: MIT Press.

Nersessian, N. J. (2009b). How Do Engineering Scientists Think? Model-Based Simulation in Biomedical Engineering Research Laboratories. Topics in Cognitive Science, 1(4), 730-757. doi:10.1111/j.17568765.2009.01032.x 
Nersessian, N. J., \& Patton, C. (2009c). Model-based reasoning in interdisciplinary engineering. In A. W. M. Meijers (Ed.), Handbook of the Philosophy of Technology and Engineering Sciences (pp. 687-718).

Nersessian, N. J. (2012). Engineering Concepts: The Interplay between Concept Formation and Modeling Practices in Bioengineering Sciences. Mind, Culture, and Activity, 19(3), 222-239. doi:10.1080/10749039.2012.688232

Ong, W. J., Lin, Z. Q., \& Domen, K. (2018). Artificial Photosynthesis: Taking a Big Leap for Powering the Earth by Harnessing Solar Energy. Particle \& Particle Systems Characterization, 35(1), 4. doi:10.1002/ppsc.201700451

Pandit, A., H. de Groot, and A. Holzwarth, (eds.) (2006). Harnessing Solar Energy for the Production of Clean Fuel. White Paper by an international task force under the auspices of the European Science Foundation. ISBN 978-90-9023907-1

Radder, H. (2003). The Philosophy of Scientific Experimentation. Pittsburgh, Penn.: University of Pittsburgh Press.

Schneider, R., F. Sander, A. Górak (2003). Dynamic simulation of industrial reactive absorption processes. Chemical Engineering and Processing 42: 955-964.

Sterrett, S. G. (2002). Physical models and fundamental laws: Using one piece of the world to tell about another. Mind \& Society, 3(1), 51-66. doi:10.1007/bf02511866

Sterrett, S. G. (2006). Models of Machines and Models of Phenomena. International Studies in the Philosophy of Science, 20(1), 69-80. doi:10.1080/02698590600641024

Sterrett, S. G. (2009). Similarity and dimensional analysis. In A. Meijers (Ed.), Philosophy of technology and engineering sciences. Handbook of the philosophy of science (Vol. 9, pp. 799-823): Elsevier/NorthHolland.

Sterrett, S. G. (2014). The Morals of Model-making. Studies in History and Philosophy of Science Part A, 46, 3145. doi:https://doi.org/10.1016/j.shpsa.2013.11.006

Sterrett, S. G. (2017). Experimentation on Analogue Models. In L. Magnani \& T. Bertolotti (Eds.), Springer Handbook of Model-Based Science (pp. 857-878). Cham: Springer International Publishing.

Suppe, F. (1989). The Semantic Conception of Scientific Theories and Scientific Realism (1989) ed.). Chicago and Urbana: University of Illinois Press.

Uosaki, K., Kondo, T., Zhang, X.-Q., \& Yanagida, M. (1997). Very Efficient Visible-Light-Induced Uphill Electron Transfer at a Self-Assembled Monolayer with a Porphyrin-Ferrocene-Thiol Linked Molecule. Journal of the American Chemical Society, 119(35), 8367-8368. doi:10.1021/ja970945p

Weber, E., T.A.C. Reydon, M. Boon, W. Houkes and P.E. Vermaas (2013). The ICE-theory of Technical Functions. Metascience 22(1): 23-44. 
Westerterp, K.R., W.P.M. van Swaaij and A.A.C.M. Beenackers (1984). Chemical Reactor Design and Operation, (2nd ed.), Chichester: Wiley.

Vincenti, W. G. (1990). What Engineers Know and How They Know it: Analytical Studies from Aeronautical History. Baltimore and London: The John Hopkins University Press.

Woodward, J. F. (2011). Data and Phenomena: A Restatement and Defense. Synthese, 182(1), 165-179. doi:10.1007/s11229-009-9618-5.

\section{Biographical Note [160 words]}

Mieke Boon is full professor in Philosophy of Science in Practice at the University of Twente (The Netherlands), where she is a core teacher in the programs Philosophy of Science, Technology and Society (PSTS) and in the University College ATLAS. In 2005 she initiated the Society for Philosophy of Science in Practice (SPSP) and was member of the SPSP organizing committee until 2016. Since 2015 she is member of the European Philosophy of Science Association (EPSA) steering committee, and chair of the chamber theoretical philosophy of National Dutch Research School in Philosophy (OZSW). She studied philosophy at the University of Leiden. Her scientific background is in chemical engineering in which she received an MSc and PhD, both with honor (cum laude). In 2003-2008 and 2012-2017 she worked on personal research grants of the Dutch National Science Foundation (NWOVidi and NWO-Vici/Aspasia), aiming to develop a philosophy of science for the engineering sciences. Research Gate. 